\title{
Comparison of Low and High Frequency Partial Discharge Measurements on Stator Windings
}

\author{
G.C. Stone \& H.G. Sedding \\ Qualitrol-Iris Power, Mississauga, Canada
}

\begin{abstract}
Partial discharge (PD) testing has been used for over 60 years primarily as a method to assess condition of the stator winding insulation in motors and generators rated $6 \mathrm{kV}$ and above. More recently it has also been used by some machine manufacturers as a means of assuring the quality of the insulation on single winding elements (coils and bars). Although both on-line and off-line tests mainly use a high voltage capacitor to detect the PD, the PD measuring systems in use work either in the low frequency (LF) regime (less than about $1 \mathrm{MHz}$ ) or in the very high frequency (VHF) (30-300 $\mathrm{MHz}$ ) range. By reference to several international standards, published work as well as some experiments described in this contribution, the advantages and disadvantages of the two approaches are compared. Based on this work, it is now clear that off-line PD tests should be done in the LF range. For online tests, either method may be used, but use of the VHF method has become more widespread with machine end users, since the owners themselves can perform and interpret the results with a relatively low risk of false indications.
\end{abstract}

\section{Introduction}

Partial discharges (PD) may occur in electrical insulation systems that operate at $3.3 \mathrm{kV}$ and above. PD only occurs when gas filled voids are present within the insulation or a gas (usually air) is present on the insulation surface when there is a high electric stress [1]. If the stress is high enough, the gas will breakdown, creating a spark consisting of energetic electrons which will break molecular bonds in any organic polymer. Thus PD will age the insulation and may eventually cause failure. PD occurs in a wide variety of high voltage electrical apparatus such as transformers, gas insulated switchgear, power cables and rotating machines. Since each discharge causes a flow of charge, the PD can be detected by measuring the current pulses on the terminals of high voltage equipment. Off-line PD testing has been used as a factory test for almost 100 years on equipment such as power cables. The purpose is to detect flaws created during manufacturing that lead to air gaps or voids that can cause PD, and thus lead to insulation failure. In the past 30 years or so, owners of high voltage equipment also measure PD over time on installed equipment. Many aging processes can create voids that can lead to PD, and thus PD is often a symptom of thermal and thermo-mechanical aging processes. By monitoring the evolution of PD over time either in off-line tests or by on-line monitoring while the equipment is operating normally, equipment owners have a powerful tool for determining when maintenance or equipment replacement is needed. This paper deals with the use of PD testing in motor and generator stator windings or coils/bars only.

Unlike power cables, switchgear and transformers, off-line factory PD testing has been relatively rarely applied as a quality assurance (QA) test for new coils or windings. Only in the past 10-15 years has there been an interest in using it as a QA test [2-4]. More commonly, machine owners have been using offline and on-line PD testing to assess the condition of the stator winding insulation in order to determine if maintenance is needed. Problems such as loose coils/bars in the stator slots, contamination leading to electrical tracking and thermal aging of the insulation are easily detected $[2,5,6]$.

There are many different types of PD test equipment that have been used for coils/bars and complete stator windings. Most use a capacitor to detect the PD pulse currents in the presence of the $50 / 60 \mathrm{~Hz}$ high voltage. The instrumentation to measure the PD current pulses most commonly includes an analog to digital converter that determines the number, magnitude and phase position (with respect to the $50 / 60 \mathrm{~Hz}$ ac cycle) of the PD. However almost every type of PD detector works in a different part of the frequency spectrum. Since each partial discharge pulse is the result of a brief flow of electrons lasting only a few nanoseconds, by the Fourier transform, frequencies from $0 \mathrm{~Hz}$ up to several hundred $\mathrm{MHz}$ are created by each discharge. Thus PD can be detected in a very wide range of frequencies, and this will affect what is actually measured. This paper discusses how the frequency range affects the detection of PD in individual coils and stator windings.

\section{Partial discharge detection standards}

The main standard referred to for PD measurement is IEC 60270 [1]. This document describes the test circuits for measuring PD, and describes the process for calibrating the detected apparent $\mathrm{mV}$ magnitude into pico Coulombs (pC). Pico Coulombs, a unit of charge, is traditionally used for PD measurement since the damage to organic insulation by the discharge will be related to the number of electrons in the discharge, i.e. the charge. Since the groundwall insulation in high voltage stator windings is partly inorganic mica, this assumption may not be valid [7]. IEC 60270 normally assumes that a capacitor detects the PD, and that the test object is also a capacitance (which is not true for stator windings). IEC 60270 also suggests the measurement frequency be anywhere in the range from $50 \mathrm{kHz}$ to $1 \mathrm{MHz}$. Any frequency can be used in 
that range, although it does categorize two sub-classes of detector:

- $\quad$ narrow band detection in the $9-30 \mathrm{kHz}$ range with a center frequency anywhere between $50 \mathrm{kHz}$ and 1 $\mathrm{MHz}$

- $\quad$ wide band detection in the $100 \mathrm{kHz}$ to $900 \mathrm{kHz}$ range.

PD detection above $1 \mathrm{MHz}$ is not covered by this standard.

In 2016, IEC 62478 was published [8]. It is a complementary document to IEC 60270 , and it covers the frequency range above the 60270 document. In particular, it defines the following ranges:

- $\quad$ Low frequency, LF -below $3 \mathrm{MHz}$ (i.e. approximately the frequency range in IEC 60270)

- High frequency, $\mathrm{HF}-3$ to $30 \mathrm{MHz}$

- Very high frequency, VHF - 30 to $300 \mathrm{MHz}$

- Ultra high frequency, UHF - 300 to $3000 \mathrm{MHz}$.

In addition to detection of the PD by capacitors, this new document indicates that PD can be measured by high frequency current transformers (HFCTs) and different types of antennae. In Clause 4.3.6, the standard makes it clear that above the LF range, a direct calibration into $\mathrm{pC}$ is not feasible as has been pointed out in the past [7,9]. Instead a "sensitivity check" is performed by comparing a conventional LF PD test (measured in $\mathrm{pC}$ ) with a test in the HF, VHF or UHF range (measured in $\mathrm{mV}$ ). The document identifies several practical schemes for measuring PD above the LF range on switchgear, transformers and stator windings.

\section{Off-line PD testing}

IEC 60270 and IEC 62478 are standards that apply to any type of high voltage equipment. IEC has also prepared two documents specifically for the stator windings and coils/bars in motors and generators. IEC 60034-27 discusses the off-line PD testing of coils/bars and complete stator windings [2]. The document, which was prepared before the publication of IEC 62478 , suggests that for both coils/bars and complete windings, the PD test be done in the IEC 60270 frequency range (i.e. below $1 \mathrm{MHz}$ ) and with a wideband detector (to minimize superposition effects).

Stator bars and coils are mainly capacitive in nature. Thus the LF range will enable the $\mathrm{PD}$ to be measured in $\mathrm{pC}$ in tests on stator coils and bars. Theoretically this implies the same PD level in $\mathrm{pC}$ should be measured on a coil or bar, independent of the specific frequency range and manufacturer of the PD detector. In practice there may be as much as a three times difference in the PD magnitude in $\mathrm{pC}$ on the same test object $[3,10]$, presumably due to different frequency ranges and detector impedances, even though they are in the LF range.

Complete stator windings are both capacitive and inductive in nature. This gives rise to a complex set of resonant frequencies in the winding. As a result, with the IEC 60270 calibration process, different calibration factors ( $\mathrm{pC}$ per $\mathrm{mV}$ ) are measured at different frequencies. As much as a 30 to 1 difference in $\mathrm{pC}$ magnitude can occur on the same winding, depending on if there is a winding resonant frequency which matches the PD detector frequency range [7]. It is for this reason that IEC 60034-27 Clauses 5.2 and 8.1 say a measurement in $\mathrm{pC}$ on a complete winding is formally not possible, and thus the PD magnitude (whether measured in $\mathrm{pC}$ or $\mathrm{mV}$ ) is a relative indicator of PD magnitude. Therefore, the PD magnitude can be only used as a comparison test, for example comparing the PD magnitude on stators of exactly the same design using the same detector and frequency range. Alternatively, if the same detector and frequency range is used, then the trend in PD magnitude over time can be measured. According to the standards, it makes no technical sense to state that $10,000 \mathrm{pC}$ measured on a $100 \mathrm{MW}$ generator is less severe than $50,000 \mathrm{pC}$ on a $200 \mathrm{MW}$ generator, even if the same PD detector is used. This is because the resonant frequencies in the 100 and $200 \mathrm{MW}$ machines are likely to be completely different, and thus trigger different responses from the PD detector.

IEC 60034-27 does indicate that it is best to measure the PD in off-line tests on a complete stator winding in the LF range. This is because in an off-line test, all the coils are energized to the test voltage, and thus any coil in the stator can have PD. Due to the inductive - capacitive nature of the stator winding, if a PD pulse occurs, for example at the neutral end of the winding, and the PD sensor is located at the phase-end of the winding, the PD pulse is likely to be attenuated and distorted as it propagates from the neutral end to the PD sensor's location [11]. The higher the detection frequency, the greater is the attenuation effect [2]. Thus PD detection in the LF range normally produces less attenuation, and one is more likely to detect PD far from the PD sensor.

\section{On-line PD testing}

In 2012, IEC 60034-27-2 was issued. It discusses on-line PD measurement in motors and generators, that is, during normal operation of the machine [5]. This document identifies the different types of sensors that can be used for on-line PD detection (coupling capacitors of different capacitances, HFCTs, and UHF antennae). There is also an extensive discussion on the need for sensor reliability, since if a PD detection capacitor fails, it will cause a forced machine outage. However, unlike for off-line testing it is noted that any frequency range (LF, HF, VHF and UHF) can be used, and has been used. Generally, from 1950 to 1980, only LF PD detection was employed, typically with a $1 \mathrm{nF}$ capacitor on each phase or an HFCT at the winding neutral. However, the signals measured not only contained stator winding PD, but also high frequency electrical signals from noise and disturbance sources - such as transmission line corona, sparking electrical contacts, slip ring sparking, variable speed drive invertor switching noise, etc. This made the extraction of the stator winding PD from the electrical interference difficult. Usually only experts with several years of experience could make reliable diagnoses [12]. As a result, since the 1980s, various researchers have explored performing on-line PD measurements at higher frequencies (not only in machines, but also in gas-insulated switchgear, cables and transformers). 
The advantage of PD detection at the higher frequencies was that better methods of suppressing noise and disturbance were possible [8]. For example, the time of flight method of separating stator PD from power system noise can be implemented if two sensors are installed per phase on the bus or cable connection between the power system and the machine (IEC 60034-27-2, Figure 1) [5,6]. Alternatively, researchers have noted that the shape of the pulse is different between stator PD and most types of power system noise. This has enabled noise and PD separation on the basis of time domain pulse shape analysis $[5,6]$ or using a software-based technique called time-frequency maps [5]. All three of these methods require the pulse arrival time to be measured with a time-domain accuracy of a few nanoseconds, and the pulse shape be recorded in the VHF range. If the signals were acquired in the LF range, the relative time of pulse arrival from two sensors cannot be distinguished, nor can the pulse shape. Another method uses UHF antennae within the generator. In the UHF range it is almost impossible for noise signals outside of the machine to penetrate within the frame, compared to LF or even VHF noise signals. Thus UHF sensors seem to provide the greatest amount of noise immunity [5, 6, 13].

The limitation of on-line VHF and UHF PD detection is that PD originating in the stator winding physically far from the PD sensor is usually more strongly attenuated than PD close to the sensor. Thus all the IEC standards caution users that PD remote from the PD sensors may not be detected in the VHF and UHF ranges. Reference [11] suggests that the VHF methods may detect PD in only $10-15 \%$ of the winding. Thus it is important to install any sensors as close as possible to the coils/bars operating at the highest voltages which are most likely to have PD, rather than near bars or coils operating at lower voltages.

\section{Comparison of PD measured at different frequency ranges}

To illustrate the differences between PD detectors using different frequency ranges, off-line PD was measured on two stator windings, with the PD measured at about the same time using different frequency ranges.

\subsection{LF vs HF off-line PD - motor stator winding}

Off-line PD tests were performed using LF and VHF PD detectors on a $13.2 \mathrm{kV} 6000 \mathrm{HP}$ motor stator winding. The LF test was performed with a $1000 \mathrm{pF}$ PD coupling capacitor. It operated in the wideband mode in frequency range $(40-800$ $\mathrm{kHz}$ ). C phase had the highest activity and is shown in Fig. 1. Fig 1(a) shows the phase resolved PD (PRPD) pattern obtained after stabilization at $8 \mathrm{kV}$ (just above rated line to ground voltage). Fig 1(b) shows the VHF PRPD plot measured on the same phase of the stator winding at the same voltage using an 80 pF PD sensor with a detection frequency range of $40-350 \mathrm{MHz}$.

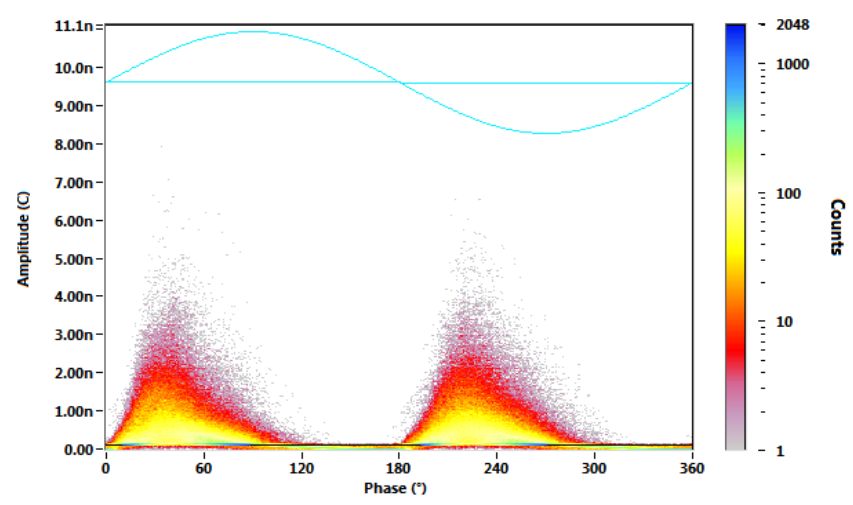

Figure 1(a)

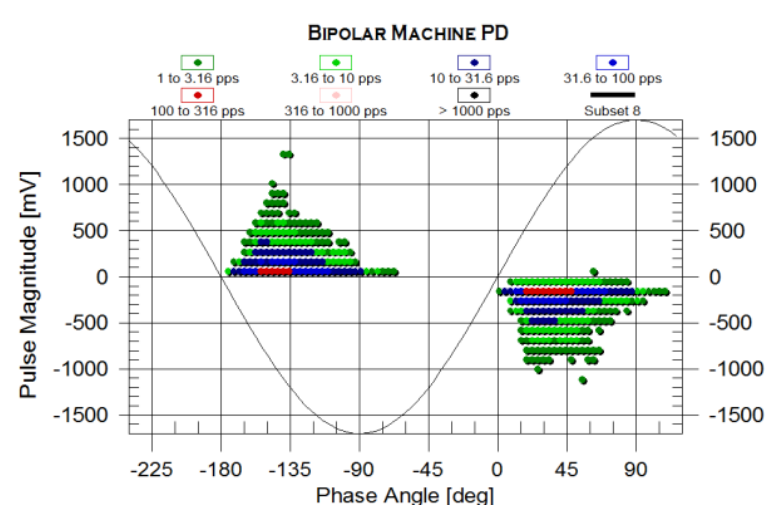

Figure 1(b)

Figure 1 : Off-line PD test on a stator winding measured in the LF (a) and VHF (b) frequency ranges. The vertical scale is the pulse magnitude in either $\mathrm{nC}$ (a) or $\mathrm{mV}$ (b). The horizontal scale is the phase angle of the $60 \mathrm{~Hz}$ AC cycle. The colour of the dots represents the number of PD pulses per second. Note that the VHF PRPD plot shows the polarity of the PD pulses. In the LF PRPD plot the pulse polarity is suppressed, but can be inferred from its position on the AC cycle.

The peak PD magnitude $(\mathrm{Qm})$ for the VHF measurement (calculated using the definition for digital instruments in IEC 60034-27) is $+816 \mathrm{mV}$ and $-912 \mathrm{mV}$. This Qm is the magnitude at a PD pulse repetition rate of 10 pulses per second (pps). Consistent with IEC 60270, where an analog definition of the peak PD magnitude is used (largest repetitive magnitude in a train of pulses), the peak PD magnitude with the LF detector is $2.5 \mathrm{nC}$. Note that different types of LF detectors implement the analog definition of Qm differently, and this may be one of the reasons for different LF PD detectors recording different values of Qm.

Using the two above definitions for the peak PD magnitude, for the $\mathrm{C}$ phase of this stator winding there is a ratio of $2.7 \mathrm{pC} / \mathrm{mV}$ between the LF and the VHF detectors. However, note the cautions in IEC 60034-27 and several papers [2-4, 7,9] that measurements with other types of LF PD instruments are likely to give much different $\mathrm{pC}$ levels. The PRPD patterns and the relationship between positive and negative $\mathrm{PD}$ is essentially the same between LF and VHF. 


\subsection{LF vs HF off-line PD - turbine generator stator}

The stator winding of an $18 \mathrm{kV}, 200 \mathrm{MVA}$ hydrogen-cooled generator was also subject to an off-line PD test using both LF and HF instruments described above. The tests were done at about $9.7 \mathrm{kV}$, a little below the rated line to ground voltage, in atmospheric pressure air. Fig 2(a) shows the PRPD plot from Phase C, which had the highest activity. The plot shows classic internal groundwall activity (see IEC 60034-27 for PRPD plots associated with each PD source), with approximately equal positive and negative PD activity. Using the IEC 60270 definition of peak PD magnitude, a Qm of $1.1 \mathrm{nC}$ was measured.

f

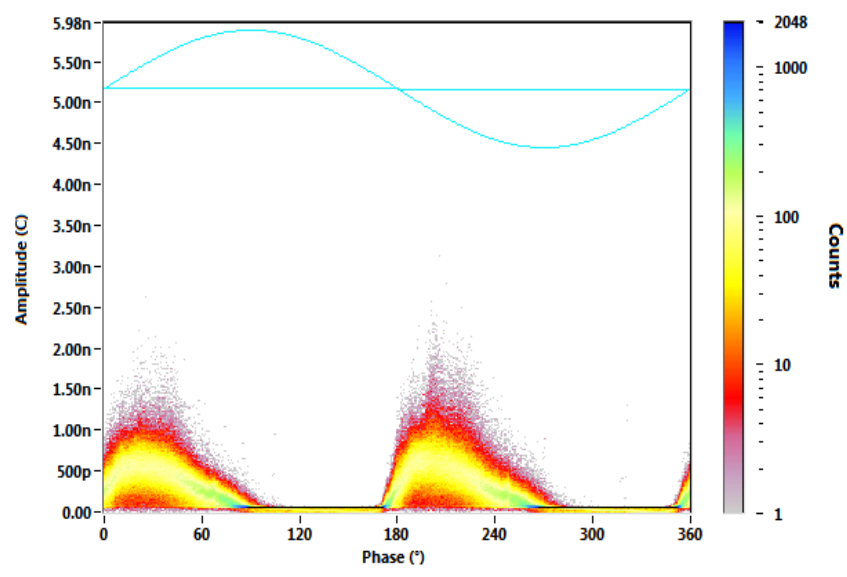

Figure 2(a)

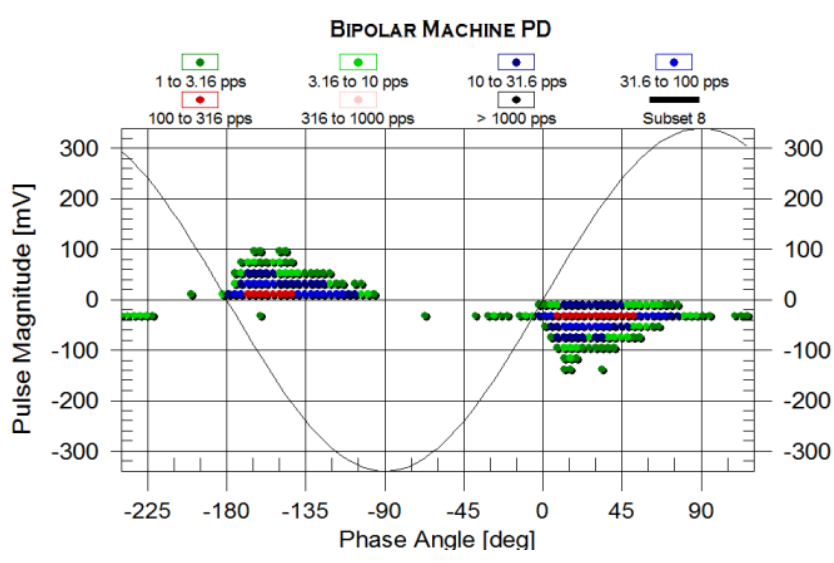

Figure 2(b)

Fig 2: LF (a) and VHF (b) off-line PD test on a 200 MVA generator stator

Fig. 2b shows the PDPD plot measured in the VHF range on $\mathrm{C}$ phase under the same test conditions as for the LF test. It shows the same PRPD pattern as for the LF test. The Qm (calculated at $10 \mathrm{pps}$ ) is $+106 \mathrm{mV}$ and $-121 \mathrm{mV}$. Thus the ratio between the LF test in $\mathrm{pC}$ and the VHF test in $\mathrm{mV}$ is $9.1 \mathrm{pC} / \mathrm{mV}$, which is substantially different than the ratio measured on the motor.

\section{Advantages of LF \& HF PD detection}

Off-line tests on both coils/bars and complete stator windings should be performed in the LF range, even though Figures 1 and 2 show the PRPD patterns are essentially the same. For coil/bar tests this will allow the PD to be quantified in terms of apparent charge $(\mathrm{pC})$. For windings, the LF range maximizes sensitivity to $\mathrm{PD}$ in more of the coils/bars in the winding.

The choice between LF and VHF (or UHF) is more difficult for on-line testing. The basic advantages of the LF test for off-line testing, as described in IEC standards [5,8], include:

- Greater sensitivity to PD occurring in coils that are further away (in space or in the circuit) from the PD sensor. This advantage is perhaps less important than for off-line tests, since the coil/bar voltage decreases linearly through the circuit from the phase terminal to the neutral end of the winding. As the voltage deceases so does the PD magnitude and the number of defects that produce PD.

- The same instrumentation used for off-line tests can be used for on-line testing, reducing instrumentation costs.

- Usually only a single sensor per phase (3 per machine) is needed for LF testing.

In contrast, the VHF and UHF tests have different advantages:

- There is greater immunity to noise and disturbances from the power system with VHF and UHF methods, which lowers the risk of false indications of stator winding problems. Also, the reduction in electrical noise, implies that less expertise is needed to perform and interpret PD results, since there is a lower risk the stator PD is obscured by the noise. This implies a lower marginal test cost. As a consequence, continuous PD monitoring is less likely to give false indications.

- With some of the VHF methods and all of the UHF methods, it is possible to locate with more certainty where the PD is occurring within the winding.

- Most capacitive PD sensors in VHF methods can meet the sensor reliability requirements stipulated in IEC 60034-27-2, thus reducing the risk that a PD sensor may fail the machine

- Peer-reviewed databases containing hundreds of thousands of test results have been summarized in tables of "high" and "low" PD. These severity levels have been confirmed by visual inspections on many hundreds of machines [14].

It is clear from the IEC standards that both the LF and VHF ranges can detect severe PD in a winding. The LF method tends to be preferred by OEMs and test service providers who have the expertise to separate the PD from the noise, and judge the severity based on experience with similar machines. Owners of machines tend to use the VHF and UHF methods since nonspecialists can perform the test and do a basic interpretation with relatively little training and experience. 


\section{Conclusions}

Side by side off-line PD tests have shown that PD can be detected in the $100-900 \mathrm{kHz}(\mathrm{LF})$ range as well as the $40-350$ $\mathrm{MHz}$ (VHF) range. The standards suggest that the LF range is used for off-line PD tests on coils and windings. However, the choice of LF vs VHF testing is not so clear for on-line PD testing of motors and generators. LF may detect more PD in the winding, but at the cost of a much greater risk of false indications when compared to VHF testing.

\section{References}

[1] IEC 60270, High Voltage Test Techniques. Partial Discharge Measurements., 2015.

[2] IEC TS 60034-27, Off-line partial discharge measurements on the stator winding insulation of rotating electrical machines, 2006.

[3] A. Petit, "Comparison of PD amplitudes of stator bars taken with different instruments", Proc IEEE Electrical Insulation Conference, June 2015, pp. 255-261.

[4] M.J. da Silva, et al, "On the Variability of PD Results Among Expert Independent Laboratories", Proc IEEE Electrical Insulation Conference, June 2016, pp194-197.

[5] IEC TS 60034-27-2, On-line partial discharge measurements on the stator winding insulation of rotating electrical machines, 2012.

[6] G.C. Stone, V. Warren, "Objective Methods to Interpret Partial-Discharge Data on Rotating-Machine Stator Windings" IEEE Transactions on Industry Applications. Vol.42, No.1, January/February 2006, pp 195-200.

[7] G. C. Stone, "Calibration of PD Measurements for Motor and Generator Windings--Why It Can't Be Done," IEEE Electrical Insulation Magazine, 9-12, January 1998.

[8] IEC 62478, High voltage test techniques - Measurement of partial discharges by electromagnetic and acoustic methods, 2016

[9] I. Kemp, "Calibration Difficulties Associated with PD Detectors in Rotating Machines", Proc IEEE Electrical Insulation Conference, October 1987.

[10] S. U1 Haq, M. Stranges \& B. Wood, “Comparative Study of IEC 60270 Compliant Instruments for PD Pattern Acquisition”, IEEE PCIC, Sept 2016.

[11] M. Henriksen, G.C. Stone \& M. Kurtz, "Propagation of PD and Noise Pulses in Turbine Generators", IEEE Trans EC, Sept 1986, pp161-166.

[12] G.C. Stone, “A Perspective on Online Partial Discharge Monitoring for Assessment of the Condition of Rotating Machine Stator Winding Insulation", IEEE Electrical Insulation Magazine, Sept 2012, pp 8-12.

[13] G.C. Stone, C. Chan \& H.G. Sedding, "Relative ability of UHF antenna and VHF capacitor methods to detect partial discharge in turbine generator stator windings", IEEE Trans DEI, Dec 2015, pp 3069-3078.

[14] H.G. Sedding, G.C. Stone \& V. Warren, "Progress In Interpreting On-Line Partial Discharge Test Results From Motor And Generator Stator Windings", Paper A1 - 202, CIGRE General Session, Paris, 2016. 